\title{
Transcriptional reprogramming strategies and miRNA-mediated regulation networks of Taxus media induced into callus cells from tissues
}

Ying Chen ${ }^{1,2,3+}$, Meng Zhang ${ }^{1,2,3+}$, Xiaofei Jin ${ }^{1,2,3}$, Haoran Tao ${ }^{1,2,3}$, Yamin Wang ${ }^{1,2,3}$, Bo Peng ${ }^{1,2,3}$, Chunhua $\mathrm{Fu}^{1,2,3^{*}}$ and Longjiang $\mathrm{Yu}^{1,2,3}$

\begin{abstract}
Background: Taxus cells are a potential sustainable and environment-friendly source of taxol, but they have low survival ratios and slow grow rates. Despite these limitations, Taxus callus cells induced through 6 months of culture contain more taxol than their parent tissues. In this work, we utilized 6-month-old Taxus media calli to investigate their regulatory mechanisms of taxol biosynthesis by applying multiomics technologies. Our results provide insights into the adaptation strategies of T. media by transcriptional reprogramming when induced into calli from parent tissues.

Results: Seven out of 12 known taxol, most of flavonoid and phenylpropanoid biosynthesis genes were significantly upregulated in callus cells relative to that in the parent tissue, thus indicating that secondary metabolism is significantly strengthened. The expression of genes involved in pathways metabolizing biological materials, such as amino acids and sugars, also dramatically increased because all nutrients are supplied from the medium. The expression level of $94.1 \%$ genes involved in photosynthesis significantly decreased. These results reveal that callus cells undergo transcriptional reprogramming and transition into heterotrophs. Interestingly, common defense and immune activities, such as "plant-pathogen interaction" and salicylic acid- and jasmonic acidsignaling transduction, were repressed in calli. Thus, it's an intelligent adaption strategy to use secondary metabolites as a cost-effective defense system. MiRNA- and degradome-sequencing results showed the involvement of a precise regulatory network in the miRNA-mediated transcriptional reprogramming of calli. MiRNAs act as direct regulators to enhance the metabolism of biological substances and repress defense activities. Given that only 17 genes of secondary metabolite biosynthesis were effectively regulated, miRNAs are likely to play intermediate roles in the biosynthesis of secondary metabolites by regulating transcriptional factors (TFs), such as ERF, WRKY, and SPL.

\footnotetext{
* Correspondence: fuch2003@126.com

†Ying Chen and Meng Zhang contributed equally to this work. 'Institute of Resource Biology and Biotechnology, Department of Biotechnology, College of Life Science and Technology, Huazhong University of Science and Technology, No.1037 Luoyu Road, Wuhan 430074, People's Republic of China

${ }^{2}$ Key Laboratory of Molecular Biophysics Ministry of Education, College of Life Science and Technology, Huazhong University of Science and Technology, No.1037 Luoyu Road, Wuhan 430074, People's Republic of China

Full list of author information is available at the end of the article
}

(c) The Author(s). 2020 Open Access This article is distributed under the terms of the Creative Commons Attribution 4.0 International License (http://creativecommons.org/licenses/by/4.0/), which permits unrestricted use, distribution, and reproduction in any medium, provided you give appropriate credit to the original author(s) and the source, provide a link to the Creative Commons license, and indicate if changes were made. The Creative Commons Public Domain Dedication waiver (http://creativecommons.org/publicdomain/zero/1.0/) applies to the data made available in this article, unless otherwise stated. 
(Continued from previous page)

Conclusion: Our results suggest that increasing the biosynthesis of taxol and other secondary metabolites is an active regulatory measure of calli to adapt to heterotrophic culture, and this alteration mainly involved direct and indirect miRNA-induced transcriptional reprogramming. These results expand our understanding of the relationships among the metabolism of biological substances, the biosynthesis of secondary metabolites, and defense systems. They also provide a series of candidate miRNAs and transcription factors for taxol biosynthesis.

Keywords: Taxus callus, miRNA, Transcription factors, Taxol biosynthesis, Plant defenses

\section{Background}

Taxol (generic name: paclitaxel), was first identified in the bark of Taxus brevifolia (Pacific yew) and is commonly used as a clinical drug for several types of cancer, such as lung cancer, and other solid tumors [1]. The substance is mainly extracted from the leaves and stems of Taxus spp., such as Taxus media. T. media is the natural hybrid of the maternal Taxus cuspidata and paternal Taxus baccata and contains considerable amounts of taxol in nearly all of its tissues [2]. However, although $T$. media has been extensively cultivated, current taxol supplies cannot meet clinical requirements due to limited land resources and the low content of the desired products in the collected plant tissues.

Induction of callus cells of Taxus spp. is considered a promising means to produce taxol and can prevent the severe misuse of conventional plant species [3]. However, most Taxus calli newly induced from tissues turn brown and cease to grow within 6-12 months, and this tissue often requires approximately 1-2 years or more to grow for industrial use [4]. Cells cultured through long-term suspension subculture show drastically reduced taxol yields and exhibit numerous other problems, such as heterogeneity and cellular ploidy [5-7]. These issues inhibit the extensive industrial use of Taxus cells for taxol production. Therefore, clarifying the regulatory mechanism of taxol biosynthesis is beneficial to address these challenges.

In a previous study, we investigated the long-term (10 years) subculture of Taxus cells and found that longterm subculture changes the living behavior of Taxus cells, resulting in reductions in taxol and other secondary metabolites and strengthening of their primary metabolism. These results indicate a vague regulatory mode in which long-term subcultured cells undergo decrease taxol biosynthesis [7].

Taxus suspension cells require approximately 1-2 years or more of subculture before they can be used for industrial culture. During the induction and formation of suspension cells, newly induced Taxus calli, which develop within 6 months of initiation of induction, contain more taxol than their parent tissues [4]. To clarify the regulatory mechanism of taxol biosynthesis in Taxus cells, we applied multiomics technology to compare callus cells with parent tissues.
Multiomics technologies have been applied to clarify complicated regulatory networks; these technologies can reveal the details of regulatory mechanisms and validate the results of high-throughput sequencing analysis [8-10]. MiRNAs have previously been recognized to be key regulators of taxol biosynthesis and transcriptional reprogramming in Taxus cells [7]. Moreover, several miRNAs regulate TFs and epigenetic factors in tissue dedifferentiation in many plants [11-13]. For example, Wu et al. found that miRNAs direct DNA methylation at loci in which they are produced, as well as in trans to their target genes, and play roles in gene regulation [14]. Shen et al. confirmed that miRNAs participate in dedifferentiation by regulating key functional genes enriched in the pathways of plant hormone signal transduction [15]. Therefore, miRNAs may be crucial factors in the regulatory mechanisms of taxol biosynthesis in callus cells.

In this work, multiomics analyses were conducted to detect the reprogrammed transcriptional profiles of newly induced callus cells, reveal the key pathways and factors influencing taxol biosynthesis and transcriptional alterations, and verify and screen key miRNAs and targets.

\section{Results}

RNA-, miRNA-, and degradome-sequencing datasets

For RNA-seq, $66.69 \mathrm{~Gb}$ clean reads were obtained from two groups through three independent biorepeats; quality control assessment showed Q20 values of 96.66\% (Table 1). Then, 74,603 unigenes with an N50 of 1464 bp were assembled, and the genes were annotated using the NR, Swiss-prot, COG, GO, and KEGG databases (Additional files 1 and 2, Table 2). Here, 11,956 unigenes were differentially expressed between the callus cells and tissues (Additional file 1d, Additional file 2, Table 2). Most differentially expressed genes (DEGs) were mainly located at the plasma membrane and the integral module of membranes (Additional file 3a, Additional file 4) and significantly involved in "plant-pathogen interaction" and "plant hormone signal transduction" (Fig. 1a, b, Additional file 5).

Through miRNA-seq, 493 miRNAs were detected, 161 of which were newly identified in $T$. media (Additional file 6). A total of 95 miRNAs, including 35 novel miRNAs, were considered to be differentially expressed 
Table 1 Quality of clean reads

\begin{tabular}{lllllllll}
\hline Sample & Raw_Reads & Raw_Bases (Gb) & Valid_Reads & Valid_Bases (Gb) & Valid\% & Q20\% & Q30\% & GC\% \\
\hline Tissue1 & $8.1 \mathrm{E}+07$ & 12.22 & $7.9 \mathrm{E}+07$ & 11.44 & 97.46 & 96.99 & 92.39 & 44.93 \\
Tissue2 & $6.8 \mathrm{E}+07$ & 10.23 & $6.7 \mathrm{E}+07$ & 9.79 & 98.25 & 98.02 & 94.76 & 44.83 \\
Tissue3 & $8 \mathrm{E}+07$ & 12.02 & $7.8 \mathrm{E}+07$ & 11.33 & 97.76 & 97.30 & 93.03 & 45.13 \\
Calli_1 & $7.4 \mathrm{E}+07$ & 11.12 & $7.2 \mathrm{E}+07$ & 10.36 & 97.41 & 96.76 & 91.80 & 46.46 \\
Calli_2 & $8.6 \mathrm{E}+07$ & 12.89 & $8.3 \mathrm{E}+07$ & 11.93 & 96.93 & 96.66 & 91.62 & 45.73 \\
Calli_3 & $8.4 \mathrm{E}+07$ & 12.56 & $8.2 \mathrm{E}+07$ & 11.84 & 97.74 & 97.33 & 93.01 & 46.27 \\
\hline
\end{tabular}

between the two groups with a $p$-value (Student T-test) of less than 0.05 (Fig. 1a, Additional files 7 and 8).

Degradome sequencing revealed that 1829 unigenes were degraded by 347 miRNAs, leading to 2432 degradation targets; of these, 323 unigenes were degraded by more than one miRNA (Additional file 9). Among the 347 identified miRNAs, cme-MIR166e-p5_2ss9CT19GC, mtr-MIR171c-p5_2ss1TC17GC, and ath-miR5021_R-1_ 1ss1TA degraded the most targets, specifically, 163, 93, and 84 unigenes, respectively. Among the degraded targets, two SPL-like TFs were degraded by the largest number of miRNAs, up to 11 (Additional file 9). SPLs (SQUAMOSA promoter-binding protein-like) form a plant-specific transcription factor family and participate in key activities; IPA1, for example, participates in the formation of plant architecture [16]. SPLs have recently been found to be tightly regulated by miRNAs in many plants, which indicates that they are crucial targets of miRNA and important nodes in the regulatory networks of plants [16-19].

Degraded differentially expressed (DE) targets were detected in approximately all DE pathways and significantly involved in 19 pathways; this result suggests that miRNAs are regulators involved in the transcriptional reprogramming of callus cells (Fig. 2a, Additional file 10).

\section{Callus cells are highly active in taxol biosynthesis but indirectly regulated by targeting miRNAs}

The taxol content of newly induced callus cells was 1.34 $\mathrm{mg} / \mathrm{gDW}$ (dry weight), which is 2.32 times higher than that in their parent tissues, and most biosynthesis genes were upregulated (Fig. 3). Additionally, the amount of 10-deacetylbaccatin III, an intermediate precursor of taxol, in callus cells was $1.02 \mathrm{mg} / \mathrm{gDW}$; such content is 3.15-fold higher than the content of parent tissues. The contents of two other taxanes in callus cells, namely, 10deacetyl taxol and baccatin III, were also high (Fig. 3b).
Taxol biosynthesis genes were found to be active, and 7 out of 12 known taxol biosynthesis genes were significantly upregulated in callus cells (Fig. 3a and c). The expression of the rate-limiting gene, 10-deacetylbaccatin III-10-O-acetyl transferase (DBAT), which was barely expressed in tissues, increased by 70.9 times in callus cells. The expression of Taxadiene synthase (TASY), which is involved in the first step of taxol synthesis, increased by over 13.96-fold in callus cells; this gene also showed a high expression level in parent tissues. Five other genes, namely, phenylpropanoyltransferase (BAPT), taxadiene 5-alpha hydroxylase (T5H), taxane 2-alphaO-benzoyltransferase (DBBT), 5-alpha-taxadienol-10beta-hydroxylase $(\mathrm{T1OH})$, and taxadienol acetyl transferase (TAT), were significantly upregulated in callus cells (Fig. 3c). These results suggest that TASY, DBAT, BAPT, $T 5 H$, and $D B B T$ are critically important for taxol biosynthesis.

Not all biosynthesis genes were upregulated in callus cells. Phenylalanine ammonia-lyase (PAM) and taxane 2-alpha hydroxylase $(T 2 H)$ were downregulated; in particular, the former was barely detectable in callus cells (Fig. 3c). The functions of these two genes requires further elucidation.

Seven homologue genes of $T 7 H, D B B T, B A P T, T A T$, $T 10 H, T 13 H, P A M$, and DBTNBT were targeted by 10 miRNAs, none of which were differentially expressed (Fig. 3a). T7H, DBBT, and BAPT are targeted by several miRNAs [7, 20-22]. Here, for the first time, BAPT was found to be targeted by miRNAs; $T 10 H$ was targeted by miR5248 and miR397a, whereas BAPT was targeted by gma-miR6300 and the Taxus-specific miRNA PC-5p97202_13 (Fig. 4c). Moreover, PC-5p-97202_13 was identified in Taxus spp. for the first time and found to degrade 34 targets (Fig. 4c, Additional file 11).

Degradome sequencing revealed that no taxol biosynthesis genes were degraded by any miRNA; however, a homologue of $T 10 H$ was degraded by mtr-miR5248

Table 2 Quality of assemblies

\begin{tabular}{|c|c|c|c|c|c|c|c|}
\hline Index & All & GC\% & Min Length & Median Length & Max Length & Total Assembled Bases & N50 \\
\hline Transcript & 127,215 & 41.15 & 201 & 503 & 17,640 & $114,510,759$ & 1556 \\
\hline Gene & 74,603 & 41.12 & 201 & 386 & 17,640 & $58,659,419$ & 1464 \\
\hline
\end{tabular}



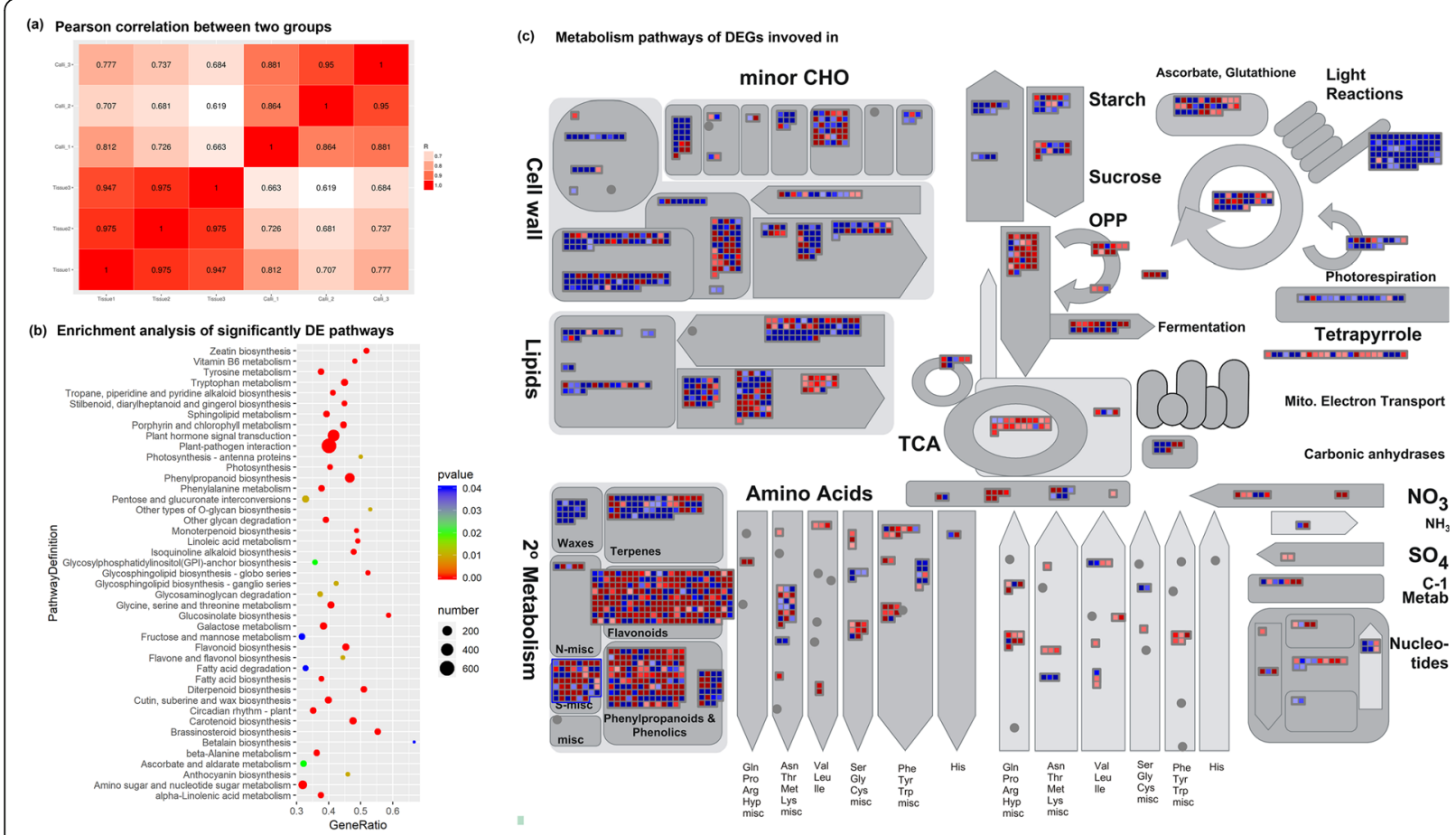

Fig. 1 Transcriptional alterations in callus cells Total six samples from two groups were high-throughput sequenced. Six samples were validated by Pearson correlation analysis (a), and they were obviously separated into two groups, callus cells and tissues. All differentially expressed genes were annotated with KEGG database, and the significant DE pathways were showed in (b). These DEGs were analyzed by Mapman3.6.0, and a metabolism overview were showed in (c)

2ss6AT21AT (Additional file 11). While T10H-like was significantly upregulated by 4.08 -fold in callus cells, the expression of mtr-miR5248_2ss6AT21AT did not differ, thus suggesting that taxol biosynthesis is not directly regulated by miRNAs in callus cells.

Taken together, these 10 miRNAs which targeted taxol biosynthesis genes degraded 226 genes that are comprehensively involved in various primary metabolic processes and common defense activities, including "planthormone signaling transduction," and "plant-pathogen interaction pathways" (Fig. 4a and b, Additional file 11).

\section{Biosynthesis of most secondary metabolites is upregulated but barely regulated by miRNAs}

Most genes involved in the biosynthesis of secondary metabolites, especially flavonoids, phenylpropanoids, lignin, and lignans, were remarkably active in callus cells (Figs. 1c and 5d). In particular, the methylerythritol phosphate (MEP) (non- mavalonic acid (MVA)) pathway, which produces terpenoid precursors, was significantly upregulated. By contrast, the MVA pathway, which is another means to produce terpenoid precursors, was downregulated (Figs. 3a and 5d). Previous reports have indicated that MEP is a highly effective and efficient means to produce terpenoid precursors and likely a positive factor for callus cells to produce additional taxanes [23].

Only $17 \mathrm{DE}$ genes of secondary metabolite biosynthesis were degraded by 15 miRNAs (Table 3). However, only five genes, namely, PER25, GT4, CYP86A22, UGT85A24, and SNL6, were upregulated because the four miRNAs that could degrade them were repressed (Table 3). Indeed, 598 DEGs involved in the biosynthesis of secondary metabolites were targeted by oppositely expressed miRNAs. These results indicate that miRNAs are capable of directly regulating secondary metabolism but they do not preferentially target metabolites in $T$. media callus cells, thus suggesting that a more costeffective regulatory system for secondary metabolism should be available.

\section{Callus cells strengthens the metabolism of biological materials and weakens the common defense system mainly mediated by miRNAs}

Among the 43 significant DE pathways found in callus cells, the pathways related to metabolism of biological substances were all strengthened (Fig. 1c, Additional file 5a, Additional file 12). For example, the metabolism of most amino acids, such as tyrosine and phenylalanine, was significantly enhanced. In addition, the DEGs of "Glycolysis" and "Citrate cycle" were remarkably 


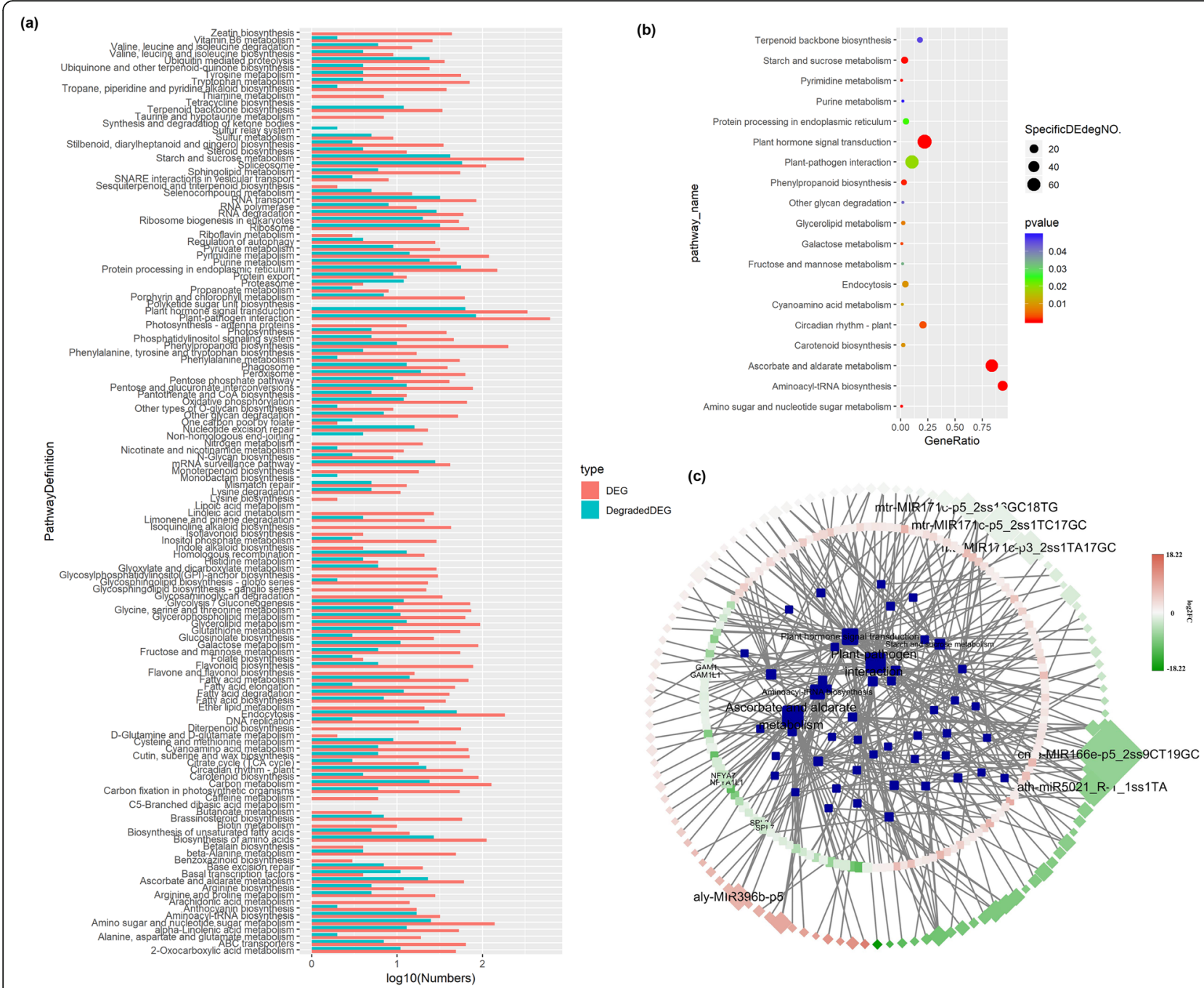

Fig. 2 Function annotation of miRNA and degraded targets. Degraded DE genes were annotated to be involved in nearly all differentially expressed pathways (a). Moreover, the DE degraded genes significantly enriched in 19 pathways (b). Among the opposite expressed miRNAs and targets, there were six miRNAs and six degraded targets found to have the most targets and be targeted respectively (c). The DE miRNAs and their DE targets mostly enriched in 5 pathways, including "Plant-Pathogen Interaction", "Plant Hormone signaling transduction", "Ascorbate and aldarate metabolism", "Starch and sucrose metabolism" and "Aminoacyl-tRNA biosynthesis". FC was short for foldchange

upregulated; both of these pathways are key processes for decomposing sugar for living energy (Fig. 1c, Additional file 5a, Additional file 12). Interestingly, "Photosynthesis" (35 downregulated vs. 3 upregulated) and "Photosynthesis-antenna proteins" (13 downregulated) were extremely weakened (Fig. 1c, Additional file 5a, Additional file 12). This finding indicates a transformation in living behavior from autotrophic tissues to heterotrophic cells.

Degradome sequencing confirmed that degraded DEGs are significantly enriched in the metabolism of several biological materials, such as "Starch and sucrose metabolism," "Ascorbate and aldarate metabolism,", and "Aminoacyl-tRNA biosynthesis" (Fig. 2b). In addition, most of the degraded DEGs involved in these pathways were regulated by oppositely expressed miRNAs, thus suggesting that miRNAs effectively regulate these pathways (Fig. 2c, Additional file 13).

Biotic and abiotic stresses are the main threats to living plants; to address these stresses, plants execute a number of response activities, such as "Plant-pathogen interaction," "Plant hormone signal transduction," "Phagosome," and "Endocytosis" [24-30]. Callus cells showed more downregulated genes involved in abiotic and biotic stress responses compared with tissues but showed upregulated heat/cold/light responses (Fig. 5a). For "Plant-pathogen interaction" and "Plant hormone signal transduction", 70\% (434) and 62\% (209) DEGs were downregulated (Fig. 5b and c, Additional file 5a). For example, jasmonic acid-amino synthetase 1 (JAR1) 


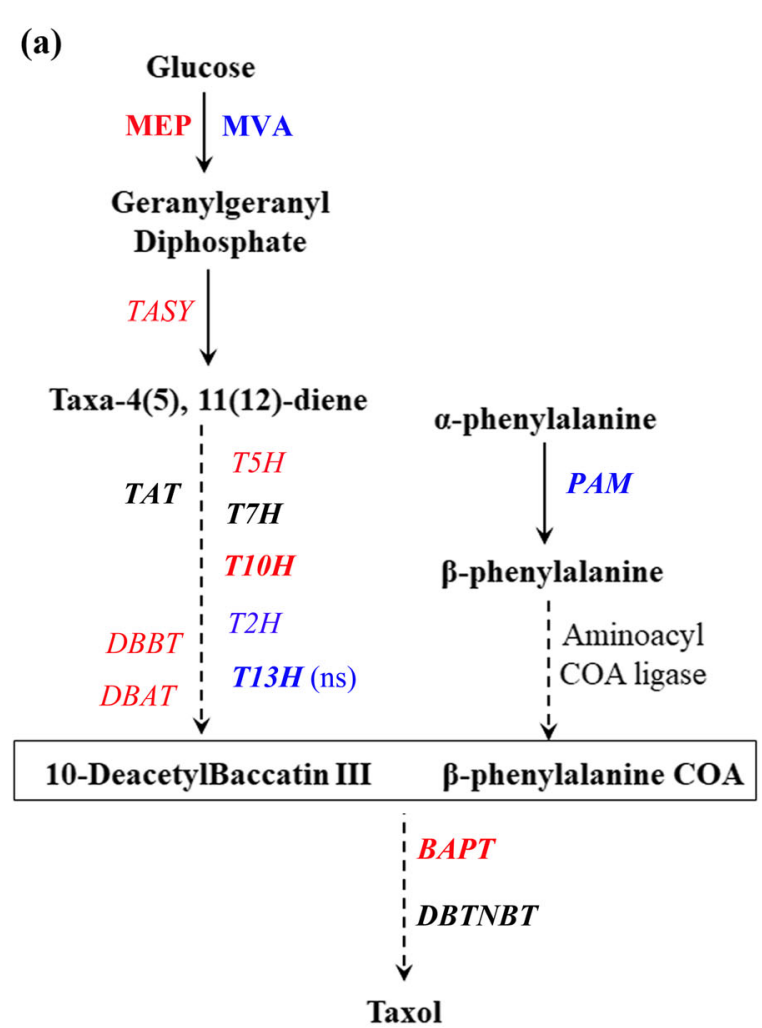

(b)

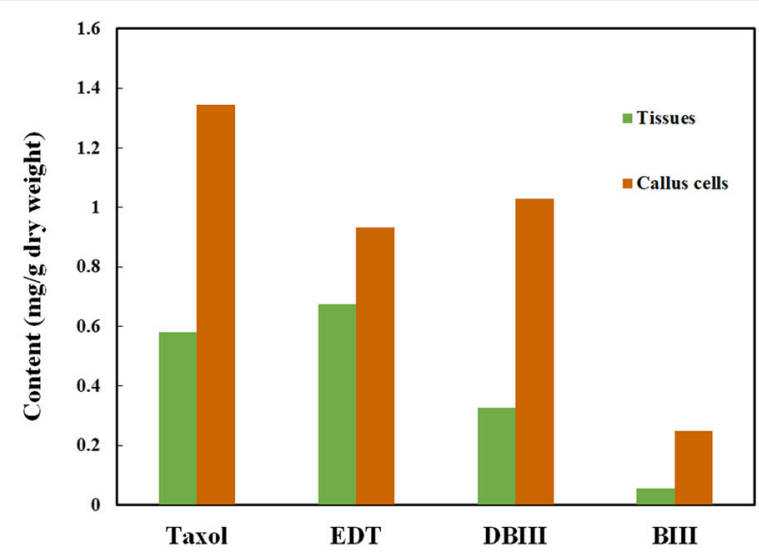

(c)

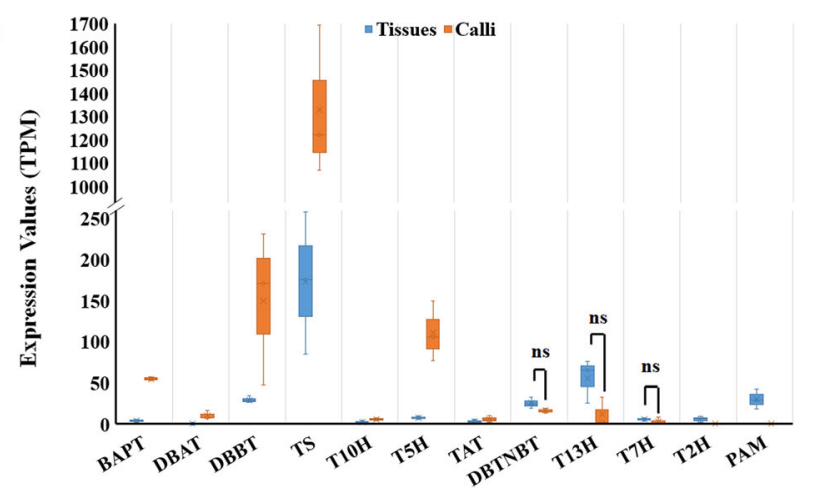

Fig. 3 Taxol biosynthesis in callus cells and tissues Taxol biosynthesis was significantly upregulated in callus cells. a Taxol biosynthesis pathways. Genes/pathways in red indicated they were upregulated, blues were downregulated, darks had no differences. And bold gens mean they were targeted by miRNAs. MEP and MVA are short for Non-mevalonate pathway and Mevalonate pathway. Solid arrows mean the enzymatic step were certificated, while dotted arrows mean there were several unknown steps. b Taxanes content in callus cells and tissues. DBIII: 10-Deacetylbaccatin III, BIII: baccatin III, EDT: 10-deacetyl taxol. c Box-plot of expression values of taxol biosynthesis genes. Ns mean the different was not significantly in callus and tissues. TS (TASY): taxadiene synthase, DBAT: 10-deacetylbaccatin III-10-0-acetyl transferaseferase, PAM: phenylalanine ammonia-lyase, T5H: taxadiene 5-alpha hydroxylase, TAT: taxadienol acetyl transferase, T10H: 5-alpha-taxadienol-10-beta-hydroxylase, T13H: 13-alpha-hydroxylase gene, DBBT: taxane 2-alpha-O-benzoyltransferase, DBTNBT: 3'-N-debenzoyltaxol N-benzoyltransferase, BAPT: phenylpropanoyltransferase

and nonexpresser of pathogenesis-related 1 (NPR1), which positively function in JA- and SA-signaling transduction, were significantly downregulated (Additional files 14 and 15). These results suggest that callus cells are fragile in terms of stress response and disease resistance.

MiRNAs are the main factors regulating common defense activities and significantly enriched in "Plantpathogen interaction" "Plant hormone signal transduction", and "Endocytosis" [31]. Among the pathways observed, "Plant-pathogen interaction" and "Plant hormone signal transduction" were the most enriched pathways regulated by miRNAs; indeed, 77 and $59 \mathrm{DE}$ degraded targets of pathogen interaction and plant hormone signal transduction were respectively detected in callus cells (Fig. 2b, Additional file 10). In addition, degraded DE targets were detected in "Phagosome", "Spliceosome" and "Base excision repair", which are other pathways related to defense. Such findings indicate that miRNA is an important regulator of these primary metabolism pathways and common defense activities. In particular, pathways, such as "plant- pathogen interaction" and "plant hormone signal transduction", are the prior regulation targets of miRNAs.

\section{TFs are important targets of miRNAs}

Among 1830 degraded targets, 635 (34.7\%) were TFs and various transcriptional regulators and degraded by 236 miRNAs, thus constituting 894 pairs of miRNA-TF modules (Additional file 9). Among the 635 degraded TFs, PHD, C3H, bHLH, WRKY, MYB, and NAC were mostly regulated by miRNAs (Fig. 6a). A total of 156 miRNAs degraded more than one TF, and cmeMIR166e-p5_2ss9CT19GC degraded 71 TFs (Fig. 6b, Additional file 16). A total of 426 miRNA-TF modules showed contrasting expression patterns, and 292 pairs (68.6\%) were constituted by downregulated miRNA and upregulated TF, thus indicating that callus cells repress the expression of miRNAs to regulate bioactivities (Additional file 16). Previous reports also concluded that miRNAs repress the expression of most genes until these genes are needed [32]. 


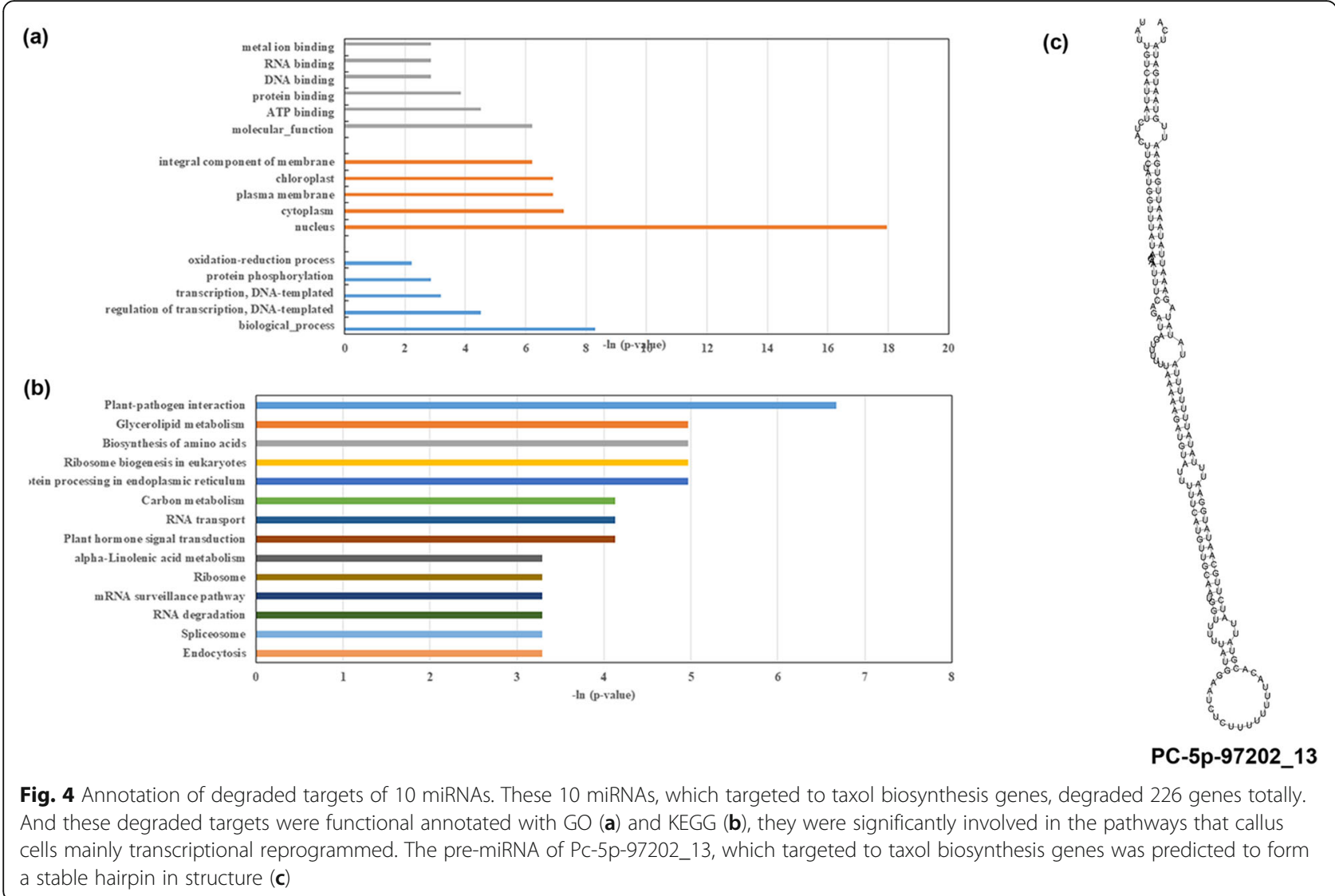

Among the TFs, ERF, bHLH, NAC, and MYB had the highest number of degraded fragments because one TF were degraded by several miRNAs, leading to a higher number of degraded fragments than DEGs (Fig. 6b). ERF, SBP, NZZ/ SPL, and NF-YA were the most enriched targets of degradation, thus suggesting their importance in transcriptional reprograming from tissues to callus cells (Fig. 6b).

The roles of these TFs were analyzed here on the basis of studies on several known Taxus TFs [33-36]. TcERF15, TcMYC2a, TcJAMYC1/2/4, and TcWRKY1/ $8 / 20 / 26 / 47 / 52$ were highly correlated with taxol biosynthesis genes with Pearson coefficients greater than 0.9 (Fig. 7b, Additional file 17). Interestingly, 4 homologues, TcMYC2a and TcJAMYC1/2/4, showed contrasting expression patterns in callus cells. These results are highly consistent with results from functional studies. TcMYC2a from Taxus chinensis and TcJAMYC1/2/4 from Taxus cuspidata function as positive and negative regulators in taxol biosynthesis, respectively [34].

\section{Candidate TFs and miRNAs involved in the regulation of taxol biosynthesis}

To determine candidate TF regulators, Pearson coexpression correlations were analyzed in combination with the previous dataset of long-term subcultured $T$. chinensis cells [7]. A total of 451 TFs were similarly or oppositely expressed with taxol biosynthesis genes (Fig. 7a), and 346 of 451 TFs were highly correlated with taxol biosynthesis genes with coefficient values greater than 0.9 (Fig. 7b, Additional file 17). $T 13 H, T 2 H$, and $P A M$ were correlated with 161, 145, and 141 TFs, respectively. Although $D B A T$ and $T 7 H$ were correlated with $107 \mathrm{TFs}$, they were simultaneously correlated with only 38 TFs (Fig. 7b, Additional file 17). TF families, NAC, WRKY, bHLH, and ERF were the most coexpressed candidate regulators.

Among the 346 miRNAs mediating TF degradation, the expression patterns of only 28 miRNAs were similar to those of taxol biosynthesis genes. Subsequent coexpression analysis confirmed that 21 miRNAs were closely related to taxol biosynthesis with high coefficient values $(>0.9$ or $<-0.9)$. Nine taxol biosynthesis genes, namely, T5H, TAT, T10H, DBBT, DBTNBT, T7H, T13H, $P A M$, and $T 2 H$, were related to miRNAs. PAM and $T 13 H$ were mainly related to 10 miRNAs; this relationship indicates that $P A M$ and $T 13 H$ are crucial regulatory targets. Hbr-MIR6173-p5_1ss9TG was coexpressed with 4 taxol biosynthesis genes. In addition, a novel miRNA first identified in Taxus, PC-5p-97202_13, simultaneously targeted $D B B T$ and $D B T N B T$; this characteristic suggests its important regulatory roles in taxol 
(a) Cellular responses

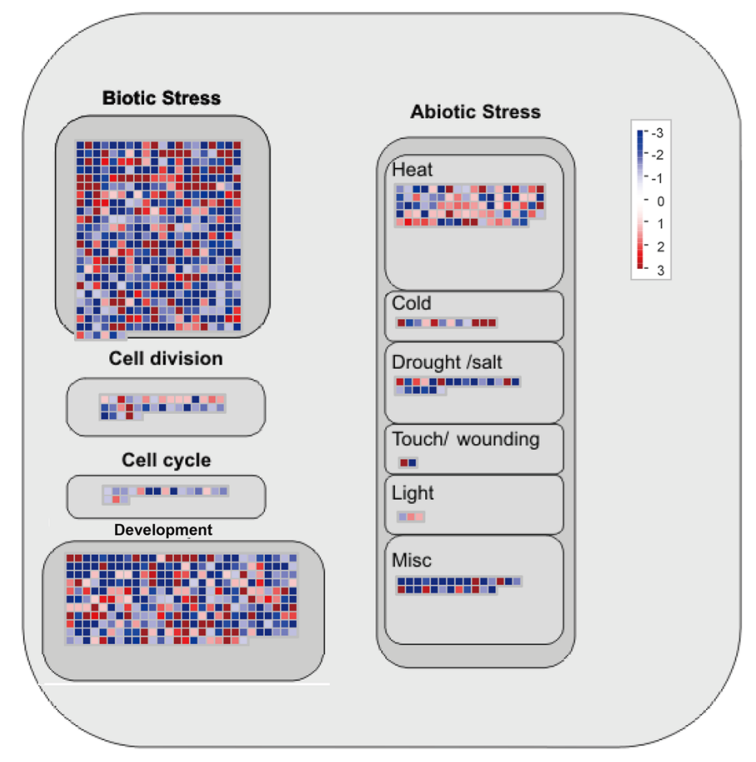

(c) Secondary metabolisms

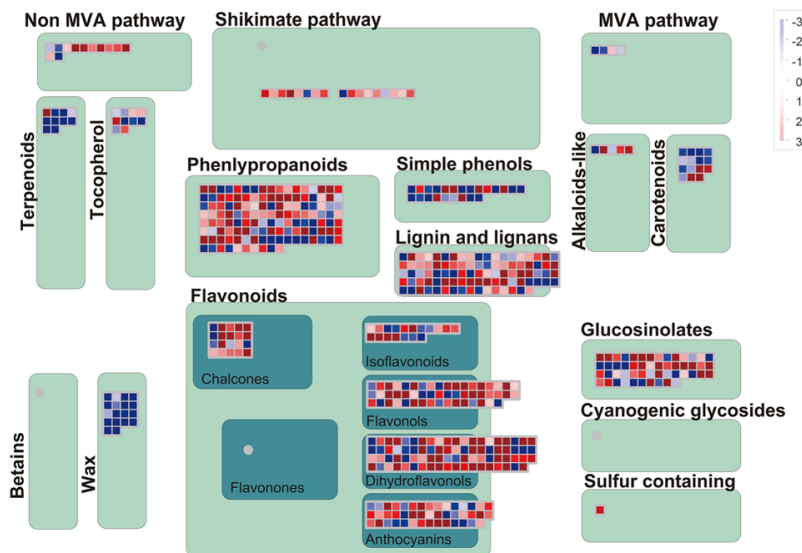

(b)

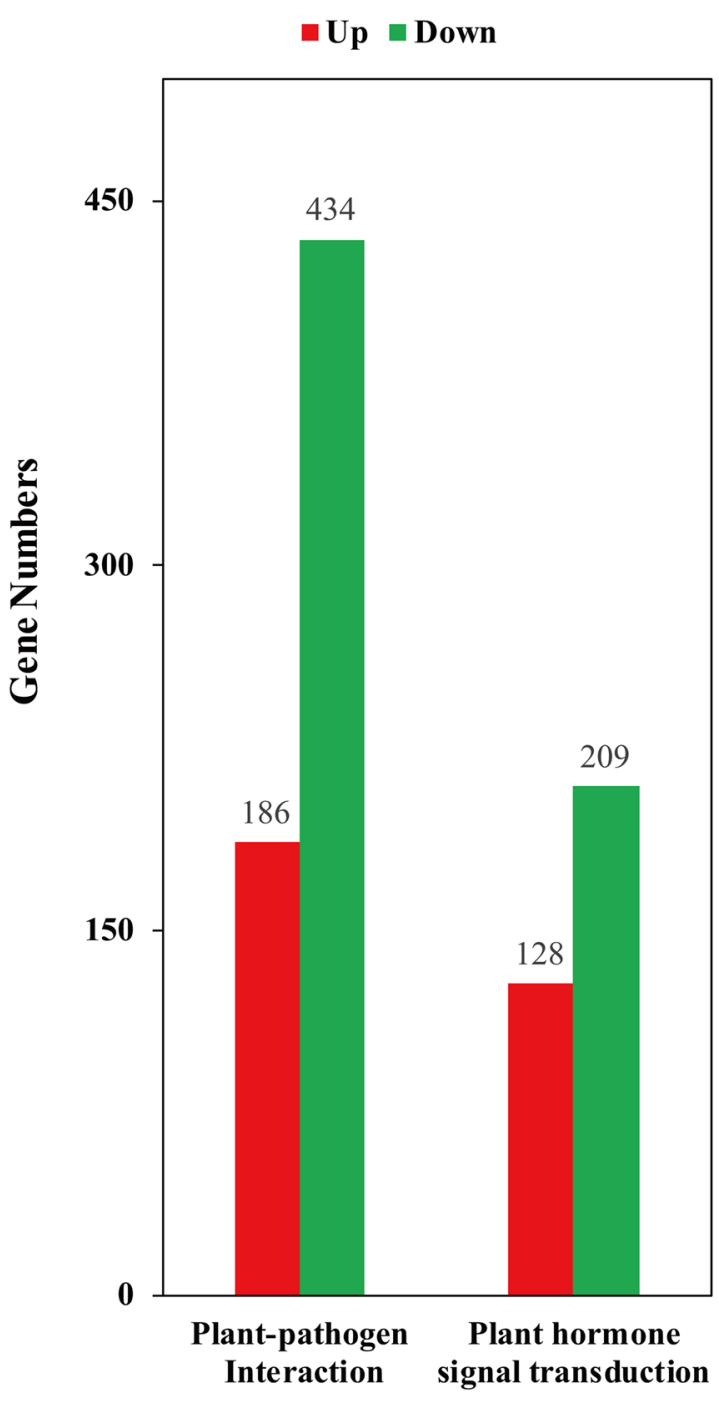

Fig. 5 Alterations of defense activities and biosynthesis of secondary metabolites in callus cells. Callus cells altered the cellular responses (a), responses of Biotic- and Abiotic-stress were dominantly changed. "Plant-pathogen interaction" and "Plant hormone signaling transduction" (b) were crucial pathways of biotic- and abiotic-stress responses, genes of them were dominantly downregulated. Biosynthesis of secondary metabolites were mostly upregulated $(\mathbf{c})$

biosynthesis (Figs. 4c, d, and 7c). Although these coexpressed miRNAs do not degrade taxol biosynthesis genes, they may regulate taxol biosynthesis through other means.

\section{Discussion}

Besides taxol, the biosynthesis of most secondary metabolites, such as flavonoids and phenylpropanoids, but not that of waxes, is remarkably enhanced in callus cells (Figs. 1c, 5d). Secondary metabolites, flavonoids, phenylpropanoids, and taxol are often used as defense materials in plants [37-39]. However, common defense activities, such as "Plant-pathogen interaction" and "Plant hormone signaling transduction", are significantly downregulated (Fig. 5, Additional file 5).

"Plant-pathogen interaction" and the JA/SA-signaling pathways help plants defend themselves against pathogens and external stimuli $[26,40]$. Our results showed that callus cells dramatically downregulate the genes of these pathways, such as JAR1 and NPR1, which are positive regulators of the JA- and SA-signaling pathways, respectively (Additional files 5 and 15). Moreover, although several pathways did not show a significant change in callus cells, they tended to be downregulated. A total of $150(81.7 \%)$ 
Table 3 Degraded DE genes involving in biosynthesis of secondary metabolites FC is short for fold change (Callus/Tissues), inf and -inf indicated they were not detected in Tissues and Callus cells respectively. AO1 (abscisic aldehyde oxidase 1), DLO2 (DMR6-like oxygenase 2), FLS (Flavonol synthase), PLR1 (Pyridoxal reductase 1), SNL6 (Cinnamoyl-CoA reductase-like SNL6), PER25 (Peroxidase 25), CBP1 (Serine carboxypeptidase 1), SDR2b (Short-chain dehydrogenase/reductase 2b) and UGT85A24 (7-deoxyloganetin glucosyltransferase)

\begin{tabular}{|c|c|c|c|c|}
\hline KEGG_pathways & miRNA & $\log 2 \mathrm{FC}(\mathrm{miRNA})$ & Gene & $\log 2 \mathrm{FC}$ (Gene) \\
\hline \multirow[t]{4}{*}{ Phenylpropanoid biosynthesis } & PC-5p-27911_99 & -2.53 & SNL6 TRINITY_DN44820_c0_g2 & 1.11 \\
\hline & $\begin{array}{l}\text { smo-MIR1083-p5 } \\
\text { 2ss1TC20GC }\end{array}$ & -3.26 & PER25 TRINITY_DN34416_c2_g2 & 6.52 \\
\hline & $\begin{array}{l}\text { nta-MIR398-p3_ } \\
\text { 2ss8AG17TC }\end{array}$ & -inf & CYP76B6 TRINITY_DN47148_C1_g1 & 4.62 \\
\hline & $\begin{array}{l}\text { ata-MIR2118b-p3 } \\
\text { 2ss8CT18AT }\end{array}$ & -inf & CBP1 TRINITY_DN42316_C1_g3 & 1.65 \\
\hline Carotenoid biosynthesis & $\begin{array}{l}\text { cme-MIR166e-p5_ } \\
\text { 2ss9CT19GC }\end{array}$ & -inf & AO1 TRINITY_DN47212_C1_g1 & 1.36 \\
\hline \multirow[t]{2}{*}{ Diterpenoid biosynthesis } & mtr-MIR5232-p5_1ss10AG & $-\inf$ & CYP71BL1 TRINITY_DN45648_C2_g1 & 1.62 \\
\hline & mtr-MIR5232-p5_1ss9TC & $-\inf$ & CYP71BL1 TRINITY_DN45648_C2_g1 & 1.62 \\
\hline \multirow[t]{3}{*}{ Flavonoid biosynthesis } & gma-MIR4995-p5 & -1.69 & DLO2 TRINITY_DN34179_c0_g1 & 8.06 \\
\hline & osa-miR529b_2ss18GT20TA & -inf & FLS TRINITY_DN45365_c0_g1 & 1.92 \\
\hline & $\begin{array}{l}\text { ptc-MIR2111a-p5 } \\
\text { 2ss9AT21GT }\end{array}$ & -0.84 & PLR1 TRINITY_DN43111_C0_g1 & 3.83 \\
\hline \multirow[t]{3}{*}{ Brassinosteroid biosynthesis } & $\begin{array}{l}\text { ath-MIR156j-p5_ } \\
\text { 2ss14CT18GT }\end{array}$ & -inf & CYP720B2 TRINITY_DN47159_C3_g1 & 6.58 \\
\hline & $\begin{array}{l}\text { sbi-MIR437w-p5 } \\
\text { 2ss4AG18AG }\end{array}$ & -0.62 & CYP720B2 TRINITY_DN40347_c0_g2 & 5.03 \\
\hline & $\begin{array}{l}\text { ath-MIR156j-p5_- } \\
\text { 2ss14CT18GT }\end{array}$ & $-\inf$ & CYP720B2 TRINITY_DN42445_CO_g2 & 2.27 \\
\hline Cutin, suberine and wax biosynthesis & PC-3p-388_5924 & 7.12 & CYP86A22 TRINITY_DN41926_C0_g1 & -5.01 \\
\hline Monoterpenoid biosynthesis & $\begin{array}{l}\text { cme-MIR166e-p5 } \\
\text { 2ss9CT19GC }\end{array}$ & -inf & SDR2b TRINITY_DN45132_c0_g1 & 2.81 \\
\hline Anthocyanin biosynthesis & $\begin{array}{l}\text { smo-MIR1083-p5_ } \\
\text { 2ss1TC20GC }\end{array}$ & -3.26 & GT4 TRINITY_DN43852_c0_g4 & 7.16 \\
\hline $\begin{array}{l}\text { Ubiquinone and other terpenoid-quinone } \\
\text { biosynthesis }\end{array}$ & ppt-MIR1030c-p5_1ss18TG & inf & At4g27270 TRINITY_DN35770_c1_g9 & -1.28 \\
\hline Benzoxazinoid biosynthesis & PC-3p-23175_128 & -1.11 & UGT85A24 TRINITY_DN45846_c2_g1 & 3.70 \\
\hline
\end{tabular}

DEGs of "Endocytosis," 32 (82.1\%) DEGs of "Phagosome," and 23 (71.8\%) DEGs of "Regulation of autophagy" were remarkably downregulated in callus cells (Additional file 5). Downregulation of these pathways indicates that callus cells become fragile in response to external stimuli. The pathways, "Nucleotide excision repair" (18 genes of 23 DEGs were downregulated), "Base excision repair" (15 down, 5 up), "Ubiquitin mediated proteolysis" (25 down, 12 up), and "Spliceosome" (76 down, 34 up) were also downregulated; this phenomenon suggests that callus cells may be susceptible to genetic mutagenesis in vivo (Additional file 5). In addition, transduction of auxin- and cytokinin-signals were inhibited because the corresponding receptors and transducing factors were downregulated, possibly explaining the slow growth rate of callus cells (Additional files 14 and 15).

According to these results of increasing of biosynthesis of secondary metabolites and reducing of defense pathways, callus cells appeared to use secondary metabolites for defense instead of common defense pathways. Given that callus cells transition to heterotrophic feeding on substances in the medium, this alteration may be related to cost-effectiveness. Specifically, "Photosynthesis" was completely downregulated in callus cells, and the metabolism of several sugars and that of almost all amino acids contained in medium were enhanced. Rojas et al. indicated that the downregulation of some primary metabolic processes, such as photosynthesis, could save energy and promote defense responses; they also revealed that the upregulation of amino acid metabolism contributes to plant defenses [37]. Moreover, secondary metabolites could be used for immediate defense materials, whereas common defense pathways, such as "Plant-pathogen Interactions" and "JA/SA signaling transduction", require time to respond. Thus, accumulation of secondary metabolites is a cost-effective strategy for survival. 


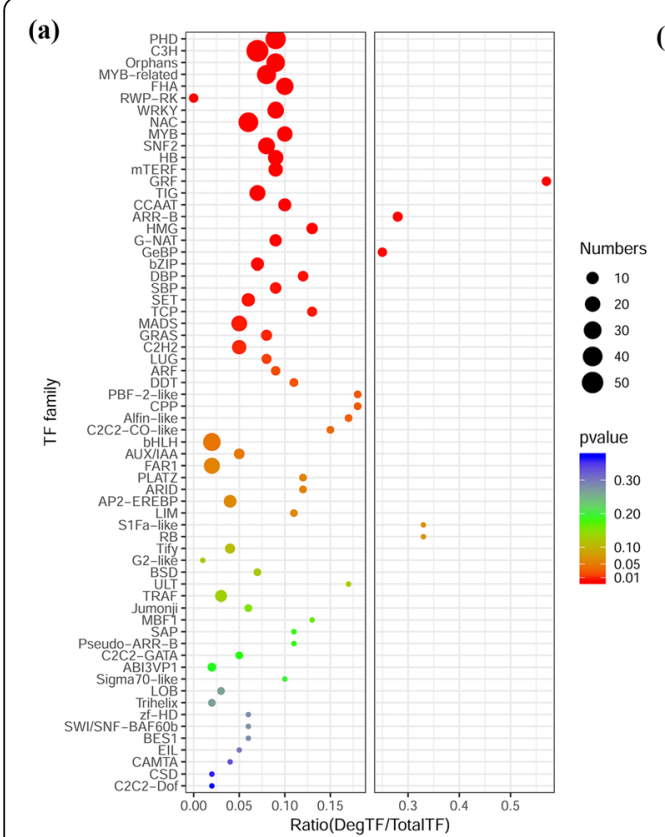

(b)

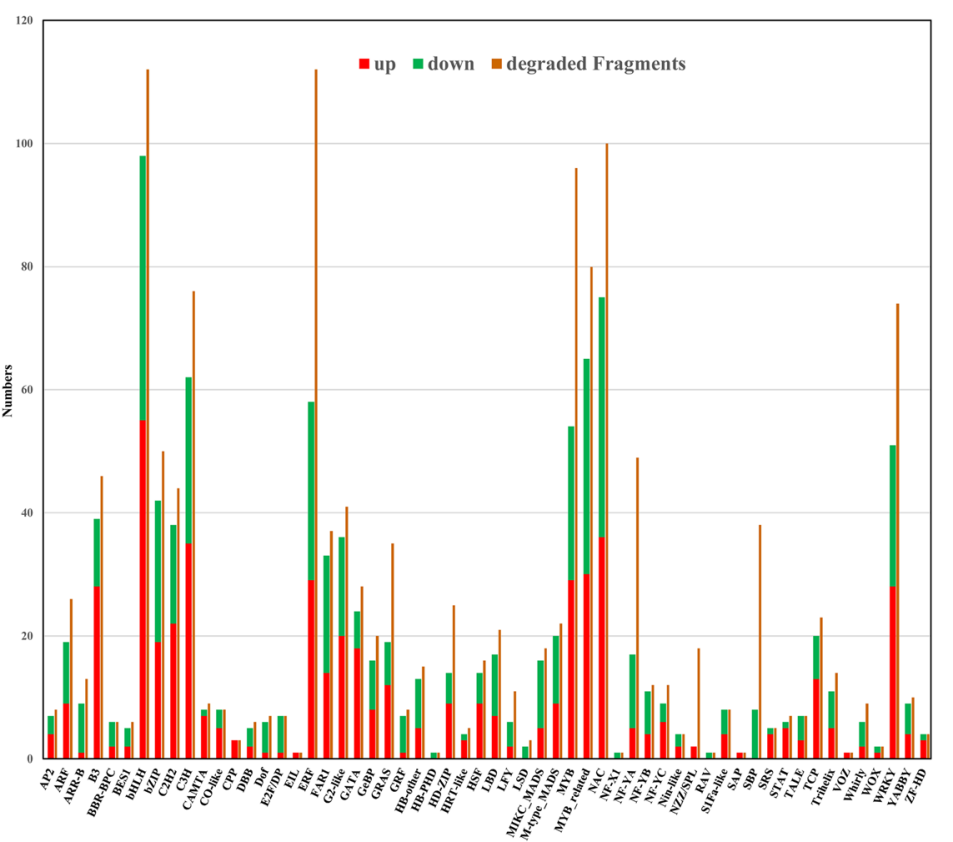

Fig. 6 Transcription factors were important targets of miRNAs. More than half of TF families were significantly degraded by miRNAs (a), and several TFs were degraded by more than one miRNAs so that degraded fragments were much more than the DE TFs, suggesting these TFs played crucial roles in transcriptional reprogramming in callus cells (b)

This inference is supported by a previous study on $T$. chinensis cells subjected to 10 years of subculture. In long-term subcultured cells, the metabolism of biological substances is enhanced, whereas common defense activities are attenuated. Remarkable reductions in taxol and other secondary metabolites suggest that long-term subculture cells abandon unnecessary defense activities [7]. These findings indicate that taxol accumulation is a survival strategy that incurs extra costs for wild plants. Thus, callus cells would prefer to choose a defense system with increased cost effectiveness.

MiRNAs are the tools used by callus cells to reprogram their metabolism. RNAs play decisive roles in changes in primary metabolism and defense systems, including biological substance metabolism, "plant-pathogen interactions", and "Endocytosis." Although miRNAs did not regulate the secondary metabolism of callus cells, they degraded a large number of TFs, such as MYB, ERF, bHLH, and other factors related to the biosynthesis of secondary metabolites. Moreover, a growing number of studies have revealed that regulation by miRNA-TFs is a main function mode in plants $[11,41,42]$.

\section{Conclusion}

Newly induced Taxus callus cells exhibited reprogramed transcriptional profiles to survive and adopt to novel situations (Fig. 8). Increasing accumulation of taxol and other secondary metabolites appeared to be an active mode to compensate for weakened immune and defense systems because callus cells repress pathways such as "Plant-pathogen interaction" and "JA/SA-signaling transduction", which are crucial for plants to defend themselves against stresses. In addition, callus cells repressed activities such as "nucleotide/protein mismatch repair" and "spliceosome" but reinforced the metabolism and biosynthesis of biological substances. These alterations suggest that callus cells prefer a cost-effective survival strategy. Subsequent analysis revealed that miRNAs are key tools for the transcriptional reprogramming of callus cells. The reduction of defense and immune systems and increased metabolism and biosynthesis of essential substances mainly depended on miRNAs. Although a small proportion of the genes involved in the biosynthesis of secondary metabolites were degraded by miRNAs, miRNAs regulated secondary metabolism by degrading a series of TFs. Several candidate TFs were highly correlated and coexpressed with taxol biosynthesis genes, and some TFs were regulated by miRNAs. Our results reveal the candidate regulators of TFs and miRNAs for taxol biosynthesis, as well as the significance of taxol biosynthesis for plant regulation networks.

\section{Methods}

\section{Plant materials and callus induction}

The 4-years-old Taxus media Rehder, obtained from Hubei green tide biotechnology company, were cultivated from cuttings at nursery in Huazhong University of Science and Technology. The tissues were fresh stems 


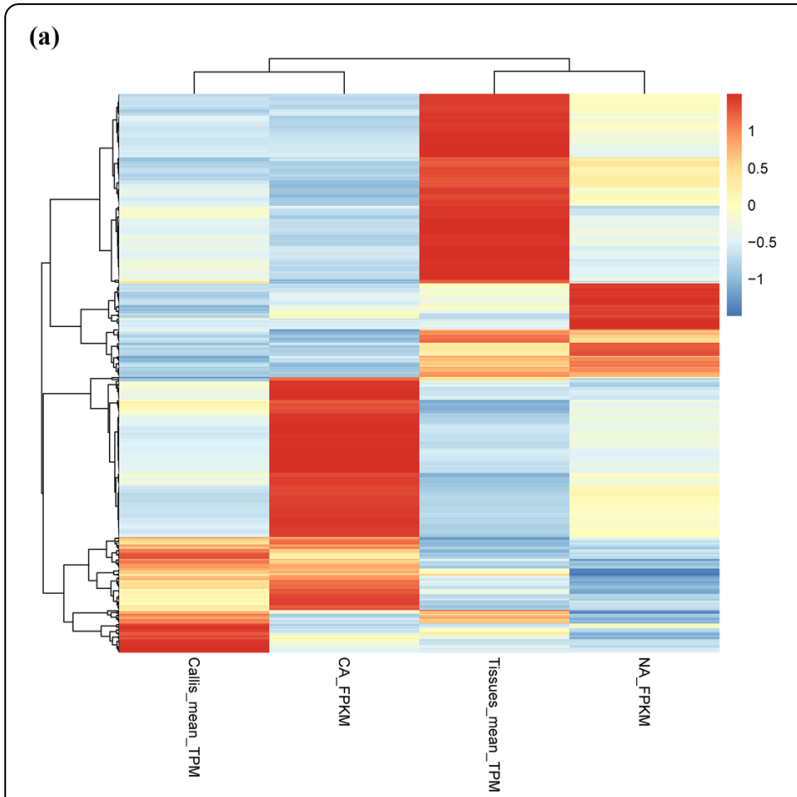

(b)
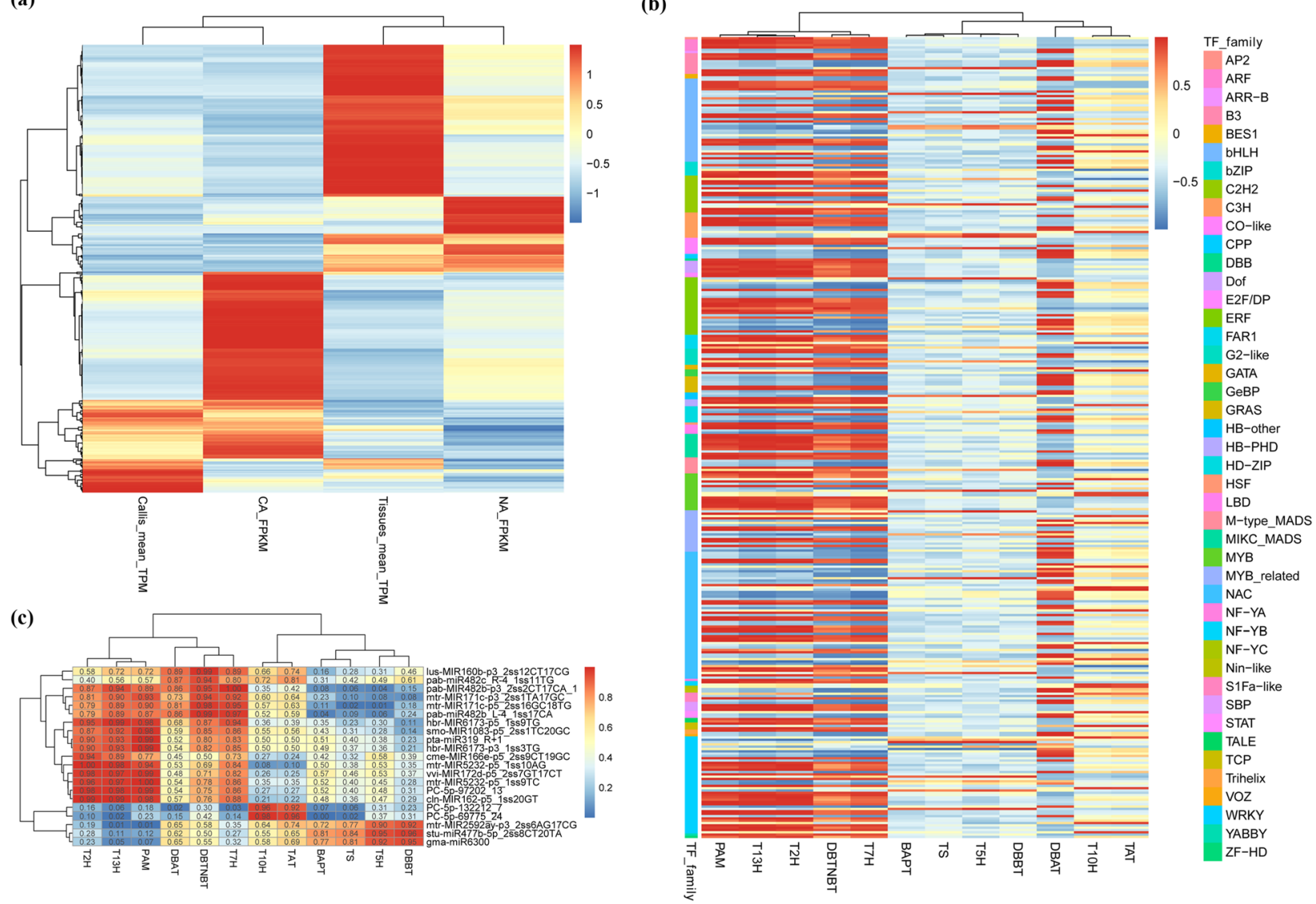

Fig. 7 Candidate TFs and miRNAs related to regulation of taxol biosynthesis. Previously, transcriptional profiles during long-term subculture process were detected. Thus, integrative analysis of these expression profiles with Callus\&Tissues were helpful to identify the key transcription factors. All DE TFs were analyzed their expression patterns comparing with 12 known taxol biosynthesis genes (a), and the co-expression relation values of candidate TFs were showed in (b). The candidate miRNAs and co-expression values were showed in (c)

and callus cells were induced with MS medium containing $0.3 \mathrm{mg} / \mathrm{L} 2,4-\mathrm{D}, 0.4 \mathrm{mg} / \mathrm{L}$ NAA from the fresh stems from the same plant, these callus cells were maintained for 6 months with 62\# (modified Gamborg's B5, the hormones concentration were $0.5 \mathrm{mg} / \mathrm{L}$ NAA, $0.5 \mathrm{mg} / \mathrm{L} 6$ $\mathrm{BA}$ and $0.2 \mathrm{mg} / \mathrm{L} 2.4-\mathrm{D})$ medium at $25^{\circ} \mathrm{C}$ without daylight [43]. Six months culturing made callus cells form a stability growth state so that it was beneficial for us to screen the key regulate factors.

\section{High-throughput mRNA sequencing, assembly and differential expression genes screening}

Total 6 samples belong to two groups (named as Tissues and Callus) were used to detect their transcriptional profiles. Total RNA isolation and library construction were conducted by LC-bio Tech (Hangzhou, China). The average insert size for the paired-end libraries was 300 bp $( \pm 50 \mathrm{bp})$, then the paired-end sequencing was performed on an Illumina Hiseq $4000(1 \times 150 \mathrm{bp}$ for each end) platform following the vendor's recommended protocol. Firstly, Cutadapter V1.9 and fqtrim V0.94 were used to remove the reads that contained adaptor contamination, low quality bases and undetermined bases [44]. Then FastQC V0.10.1 was used to verify sequence quality (http://www.bioinformatics.babraham.ac.uk/projects/fastqc/) including the Q20, Q30 and GC-content of the clean data. All downstream analyses were based on clean data of high quality, which did not contain any adaptor contamination (if the read was smaller than 100 bp after removing the adapter, the read were deleted), low quality bases (if the average quality of every $6 \mathrm{bp}$ of single read is lower than 20 , the entire 3 '-flanking sequence was deleted) and undetermined bases (if the unknown base is more than $5 \%$ in a read, the read will be deleted). De novo assembly of the transcriptome was performed with Trinity 2.4.0. All assembled Unigenes were aligned against the non-redundant $(\mathrm{Nr})$ protein database (http://www.ncbi.nlm.nih.gov/), Gene ontology (GO) (http://www.geneontology.org), SwissProt (http:// www.expasy.ch/sprot/), Kyoto Encyclopedia of Genes and Genomes (KEGG) (http://www.genome.jp/kegg/) and eggNOG (http://eggnogdb.embl.de/) databases using 


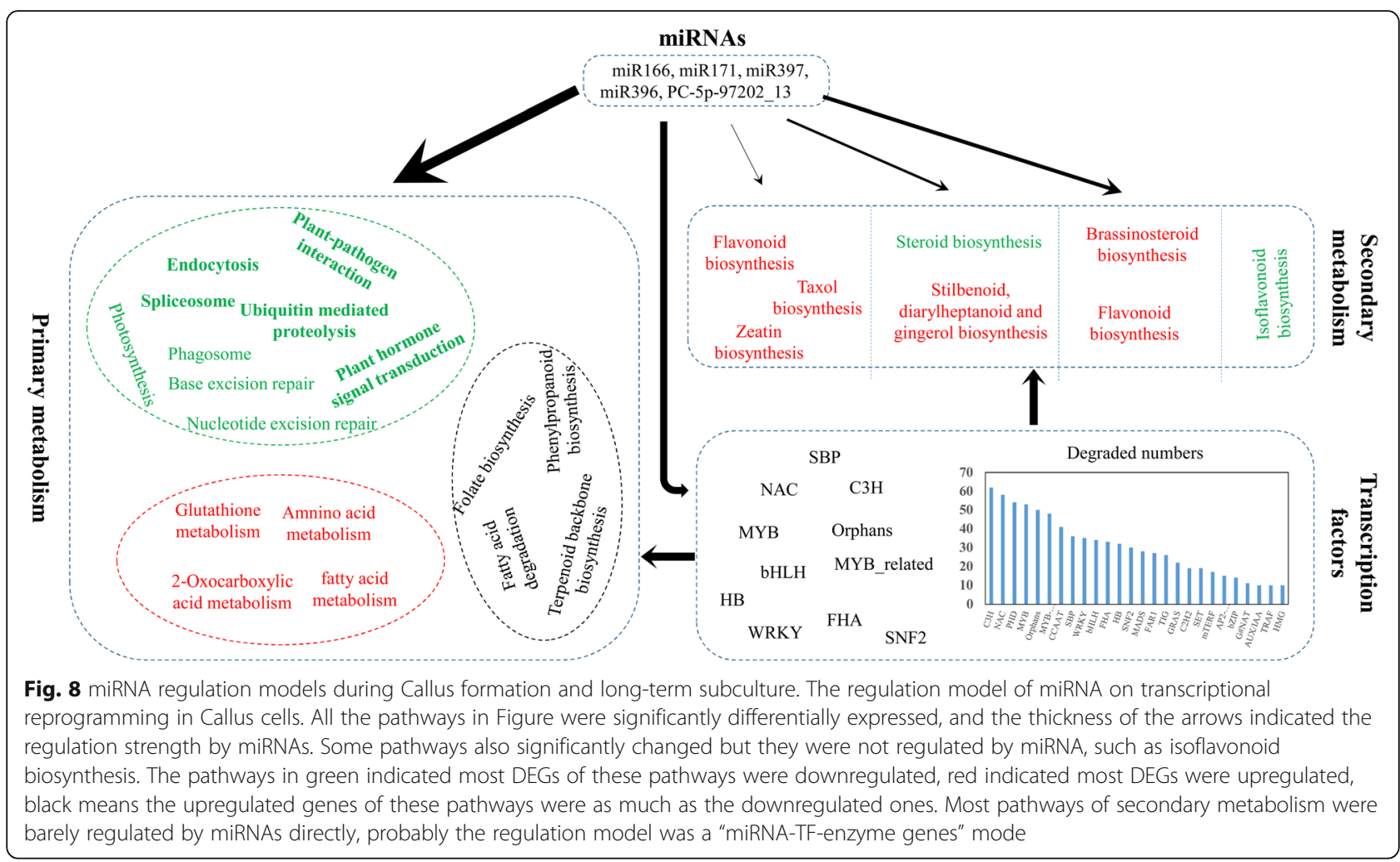

DIAMOND [45] with a threshold of E-value $<1$ e-5. Salmon was used to perform expression level for Unigenes by calculating TPM [46], and R package edgeR was applied for calculating statistical significance ( $p$-value), then FDR were used to detected the false rate [47]. Since there were three independent bio-repeats of each individual group, the differentially expressed unigenes were selected not only with $\mid \log 2$ (fold change) $\mid>1$, but also with FDR $<0.05$. The GO and KEGG enrichment analysis of DEGs were validated by Fisher's Exact Test with a cutoff $p$-value $<0.05$.

\section{MiRNA analysis and targets prediction}

Part of total RNAs from the same 6 samples of transcriptome were also used to conduct library for miRNAseq. The miRNA library was constructed with TruSeq Small RNA Sample Prep Kits (Illumina, San Diego, USA), and they were single-end sequenced by Illumina Hiseq2000/2500 with $1 \times 50$ bp. ACGT101-miR (LC Sciences, Houston, Texas, USA) was used to process raw reads by removing adapter dimers, junk (unknown nucleotide bases more than 10\%), low complexity, common RNA families (rRNA, tRNA, snRNA, snoRNA) and repeats as described by $\mathrm{Hu}$ et al., 2018 [48]. Subsequently, unique sequences with length in 18 25 nucleotide were mapped to specific species precursors in miRBase 21.0 by BLAST search to identify known miRNAs that allowed length variation at both $3^{\prime}$ and $5^{\prime}$ ends and one mismatch inside of the sequence. For novel miRNAs, the unmapped sequences were BLASTed against the shallow sequencing Taxus baccata genomes [49], and the hairpin RNA structures containing sequences were predicted from the flank $120 \mathrm{nt}$ sequences using RNAfold software (http://rna.tbi.univie.ac. at/cgi-bin/RNAfold.cgi).

Firstly, expression levels of miRNAs were based on normalized deep-sequencing counts, the normalization procedures were according to Zhao et al., 2017 [50]. Differential expression of miRNAs was analyzed by using Student T-test, when the p-value was less than 0.05 , the miRNAs were considered as differentially expressed miRNAs [50] (Additional file 18).

To predict the genes targeted by most abundant miRNAs, Target Finder ver. 50 were used to identify miRNA binding sites using default settings [51].

\section{Degradome sequencing and data processing}

Approximately $20 \mu \mathrm{g}$ total RNA that mixed from $6 \mathrm{sam}$ ples were used to prepare degradome library followed as with some modification [52]. Then, the libraries were sequenced using the 5'adapter only, resulting in the sequencing of the first 36 nucleotides of the inserts that represented the $5^{\prime}$ ends of the original RNAs. And the single-end sequencing ( $36 \mathrm{bp}$ ) were performed on an Illumina Hiseq2500.

After removing adaptors and low quality reads, the extracted sequencing reads were then used to identify 
potentially cleaved targets by the CleaveLand pipeline [53]. The degradome reads were mapped to the transcripts obtained above. Only the perfect matching alignment(s) for the given read would be kept for degradation analysis. All resulting reads (t-signature) were reverse complemented and aligned to the miRNA identified in our study. All the identified targets were subjected to BlastX analysis to search for similarity, and then to GO analysis to uncover the miRNA-gene regulatory network on the basis of biological process, cellular module and molecular function.

\section{Taxanes extraction and quantitative analysis}

The tissues and callus cells were firstly grinding with liquid Nitrogen, then taxanes were extracted using the method described previously [54]. And the quantitative analysis was conducted using HPLC as the previous reports [36].

\section{Transcription factors and transcriptional regulators identification}

Transcription factors and transcriptional regulators annotation were conducted by blasting the assembling unigenes against plnTFdb V3.0 (http://plntfdb.bio.unipotsdam.de/v3.0/) with the E-values <1e-5. Expression patterns were drawn by Morpheus (https://software. broadinstitute.org/morpheus/) with defaulting parameters, and Pearson co-expression coefficient values were analyzed by CORREL formula within Excel 2016 [55, $56]$.

\section{Supplementary information}

Supplementary information accompanies this paper at https://doi.org/10. 1186/s12864-020-6576-2

Additional file 1. Information of RNA-seq results The length distribution of assembled unigenes was showed in (a). Species distribution by annotated with NR was showed in (b), Picea sitchensis had the most homologues of Taxus media. (c) eggNOG annotation of all unigenes. (d) Expression volcano map of unigenes. (e) Expression patterns of all DEGs.

Additional file 2. Information of miRNA-seq The length distribution of miRNAs was showed in (a). Total and first nucleotide bias were showed in (b) and (c) respectively. (d) Pearson relation analysis of all expressed miRNAs between six samples. (e) Venn diagram of miRNAs in callus cells and tissues. (f) Expression patterns of all DE miRNAs $(p<0.05)$.

Additional file 3. Annotation and expression levels of assembled unigenes.

Additional file 4. All expressed miRNAs All expressed miRNAs were listed in sheet (a) including known and novel miRNAs. The sheet (footnotes) provided the information about the table.

Additional file 5. $G O$ annotations and statistics of DEGs $G O$ statistic of DEGs (a) and GO annotation of DEGs (b). The genes with only one specific annotation was counted.

Additional file 6. GO annotation of DEGs and degraded DEGs Supplement figure compared the GO annotations of DEGs ( $a$ and b) with degraded DEGs (c and d). (a) GO annotations of DEGs, (b) GO enrichment analysis of DEGs (first 20 terms). (c) GO annotations of degraded DEGs, (d) $\mathrm{GO}$ enrichment analysis of degraded DEGs (first 20 terms). All these results suggested that miRNAs comprehensively regulated in transcriptional reprogramming in callus cells.

Additional file 7. Alteration maps of several important bioactivities Several important bioactivities were listed here: (a) biotic- and abioticstress responses, (b) regulators, (c) large enzyme families, (d) Planthormone transduction and (e) overview of cell functions. These drawing were made by Mapman, and the boxes indicated genes, red means upregulated and blue was down regulated.

Additional file 8. KEGG annotations and statistics of DEGs KEGG statistic of DEGs (a) and KEGG annotation of DEGs (b). The genes with only one specific annotation was counted.

Additional file 9. DE miRNAs and their expression levels.

Additional file 10. Degradome-seq results All the degraded targets and miRNAs were listed. $Y$ in column (DegradomeDetection) means the degraded fragment was sequenced, while $\mathrm{N}$ indicated the target was only predicted by software TargetFinder.

Additional file 11. Statistics of KEGG annotations The KEGG annotations of degraded DEGs were validated along with the DEGs, and the results showed that among 52 DE KEGG pathways, 19 were significantly regulated by miRNAs.

Additional file $\mathbf{1 2}$ Taxus media mapman database annotations.

Additional file 13. Ten miRNA regulatory networks which targeted to taxol biosynthesis genes.

Additional file 14. Targets of 10 miRNAs which targeted to taxol biosynthesis genes All degraded targets of 10 miRNAs were listed.

Additional file 15. DEGs related to Plant Hormone Signaling Transduction All DEGs that related to "Plant Hormone Signaling Transduction" were listed. BR was short for Brassinosteroid, SA short for Salicylic acid, GA short for Gibberellin acid, ABA short for Abscisic acid, JA short for Jasmonic acid and ET short for Ethylene.

Additional file 16. Degraded transcription factors All degraded transcription factors and related miRNAs were listed in (a). Transcription factors were annotated with plnTFdb database.

Additional file 17. Co-expression values of TFs with taxol biosynthesis genes The co-expression correlation co-efficiency values were displayed.

Additional file 18:. miRNA norization method.

\section{Abbreviations}

AO1: Abscisic aldehyde oxidase; BAPT: Phenylpropanoyltransferase; BIII: Baccatin III; DAB III: 10-Deacetyl Baccatin III; DBAT: 10-deacetylbaccatin III10-O-acetyl transferaseferase; DBBT: Taxane 2-alpha-O-benzoyltransferase; DBTNBT: 3'-N-debenzoyltaxol N-benzoyltransferase; DLO2: DMR6-like oxygenase 2; FLS: Flavonol synthase; JA: Jasmonic acid; JAR1: Jasmonic acidamino synthetase 1; NPR1: Nonexpresser of pathogenesis-related 1; PAM: Phenylalanine ammonia-lyase; PLR1: Pyridoxal reductase 1; SA: Salicylic acid; T10H: 5-alpha-taxadienol-10-beta-hydroxylase; T13H: 13-alphahydroxylase gene; $\mathrm{T} 2 \mathrm{H}$ : Taxane 2-alpha hydroxylase; $15 \mathrm{H}$ : Taxadiene 5-alpha hydroxylase; TASY: Taxadiene synthase; TAT: Taxadienol acetyl transferase; TF: Transcription factors

\section{Acknowledgements}

Thanks for the RNA quality detection supports in Core Facilities of life sciences in College of Science and Technology, Huazhong university of science and technology. And thank the Analytical and Testing Center in Huazhong University of Science \& Technology for HPLC-MS analysis. We are also grateful to LC Sciences company (Hangzhou, China) for technical support and several data analyses. And thanks for the EnPapers for language editing.

\section{Authors' contributions}

CF and LY conceived the research, YC, MZ and CF designed the work. YC, $M Z, X J, H T, Y M$ and BP conducted the experiments, including plant cultivation, Callus cells induction and subculture, plant material harvest, taxanes content detection and so on. YC and $\mathrm{MZ}$ analyzed the data and wrote the manuscript. All authors read and approved the manuscript. 


\section{Funding}

This work was supported by Hubei Technological Innovation Special Fund (CN) (2019ABA091) The funding bodies did not play a role in the design of the study and collection, analysis, and interpretation of data and in writing the manuscript, but just provide the financial support.

\section{Availability of data and materials}

All the raw reads of mRNA-, miRNA-seq and degradome data were submitted to SRA (Sequence Read Archive, NCBI, https://www.ncbi.nlm.nih.gov/sra/) under the Bioproject number PRJNA497757, the accession numbers are SRR8083193 to SRR8083198.

\section{Ethics approval and consent to participate}

The Hubei green tide biotechnology company, provided permission to use the seedlings of T. media for our scientific research.

\section{Consent for publication}

Not applicable.

\section{Competing interests}

The authors declare that they have no conflict of interest.

\section{Author details}

Institute of Resource Biology and Biotechnology, Department of Biotechnology, College of Life Science and Technology, Huazhong University of Science and Technology, No.1037 Luoyu Road, Wuhan 430074, People's Republic of China. ${ }^{2}$ Key Laboratory of Molecular Biophysics Ministry of Education, College of Life Science and Technology, Huazhong University of Science and Technology, No.1037 Luoyu Road, Wuhan 430074, People's Republic of China. ${ }^{3}$ Hubei Engineering Research Center for Edible and Medicinal Resources, Wuhan 430074, People's Republic of China.

\section{Received: 13 January 2020 Accepted: 11 February 2020} Published online: 18 February 2020

\section{References}

1. Weaver BA. How Taxol/paclitaxel kills cancer cells. Mol Biol Cell. 2014;25(18): 2677-81.

2. Yu C, Guo H, Zhang Y, Song Y, Pi E, Yu C, Zhang L, Dong M, Zheng B, Wang $\mathrm{H}$, et al. Identification of potential genes that contributed to the variation in the taxoid contents between two Taxus species (Taxus media and Taxus mairei). Tree Physiol. 2017:37(12):1659-71.

3. Fujita Y, Hara Y, Suga C, Morimoto T. Production of shikonin derivatives by cell suspension cultures of Lithospermum erythrorhizon : II. A new medium for the production of shikonin derivatives. Plant Cell Rep. 1981;1(2):61-3.

4. Wickremesinhe ERM, Arteea RN. Taxus callus cultures: initiation, growth optimization, characterization and taxol production. Plant Cell Tissue Organ Cult. 1993;35(2):181-93.

5. Naill MC, Roberts SC. Cell cycle analysis of Taxus suspension cultures at the single cell level as an indicator of culture heterogeneity. Biotechnol Bioeng. 2005;90(4):491-500.

6. Miguel C, Marum L. An epigenetic view of plant cells cultured in vitro: somaclonal variation and beyond. J Exp Bot. 2011;62(11):3713-25.

7. Zhang M, Dong Y, Nie L, Lu M, Fu C, Yu L. High-throughput sequencing reveals miRNA effects on the primary and secondary production properties in long-term subcultured Taxus cells. Front Plant Sci. 2015;6:604

8. Li W, Jia Y, Liu F, Wang F, Fan F, Wang J, Zhu J, Xu Y, Zhong W, Yang J. Integration Analysis of Small RNA and Degradome Sequencing Reveals MicroRNAs Responsive to Dickeya zeae in Resistant Rice. Int J Mol Sci. 2019; 20(1):222. https://doi.org/10.3390/ijms20010222.

9. Hua Y, Zhang C, Shi W, Chen H. High-throughput sequencing reveals microRNAs and their targets in response to drought stress in wheat (Triticum aestivum L.). Biotechnol Equip. 2019;33:1-7.

10. Ma C, Yang J, Cheng Q, Mao A, Zhang J, Wang S, Weng Y, Wen C. Comparative analysis of miRNA and mRNA abundance in determinate cucumber by high-throughput sequencing. PLoS One. 2018;13(1):e0190691.

11. Wang L, Liu N, Wang T, Li J, Wen T, Yang X, Lindsey K, Zhang X. The GhmiR157a-GhSPL10 regulatory module controls initial cellular dedifferentiation and callus proliferation in cotton by modulating ethylenemediated flavonoid biosynthesis. J Exp Bot. 2018;69(5):1081-93.
12. Sabana AA, Antony G, Rahul CU, Rajesh MK. In silico identification of microRNAs and their targets associated with coconut embryogenic calli. Agri Gene. 2018;7:59-65.

13. Rodriguez-Enriquez J, Dickinson HG, Grant-Downton RT. MicroRNA misregulation: an overlooked factor generating somaclonal variation? Trends Plant Sci. 2011;16(5):242-8.

14. Wu L, Zhou H, Zhang Q, Zhang J, Ni F, Liu C, Qi Y. DNA methylation mediated by a microRNA pathway. Mol Cell. 2010;38(3):465-75.

15. Shen Y, Jiang Z, Lu S, Lin H, Gao S, Peng H, Yuan G, Liu L, Zhang Z, Zhao M, et al. Combined small RNA and degradome sequencing reveals microRNA regulation during immature maize embryo dedifferentiation. Biochem Biophys Res Commun. 2013;441(2):425-30.

16. Song X, Lu Z, Yu H, Shao G, Xiong J, Meng X, Jing Y, Liu G, Xiong G, Duan J, et al. IPA1 functions as a downstream transcription factor repressed by D53 in strigolactone signaling in rice. Cell Res. 2017:27(9):1128-41.

17. Wang $L$, Zhang Q. Boosting Rice yield by fine-tuning SPL gene expression. Trends Plant Sci. 2017:22(8):643-6.

18. Wang JW. The Multifaceted Roles of miR156targeted SPL Transcription Factors in Plant Developmental Transitions. Plant Transcription Factors. 2016. p. 281-93.

19. Stief A, Altmann S, Hoffmann K, Pant BD, Scheible WR, Baurle I. Arabidopsis miR156 regulates tolerance to recurring environmental stress through SPL transcription factors. Plant Cell. 2014;26(4):1792-807.

20. Sun G, Yang Y, Xie F, Wen JF, Wu J, Wilson IW, Tang Q, Liu H, Qiu D. Deep sequencing reveals transcriptome re-programming of Taxus media cells to the elicitation with methyl jasmonate. PLoS One. 2013;8(4):e62865.

21. Hao DC, Yang L, Xiao PG, Liu M. Identification of Taxus microRNAs and their targets with high-throughput sequencing and degradome analysis. Physiol Plant. 2012;146(4):388-403.

22. Qiu DY, Pan XP, Wilson IW, Li FL, Liu M, Teng WJ, Zhang BH. High throughput sequencing technology reveals that the taxoid elicitor methyl jasmonate regulates microRNA expression in Chinese yew (Taxus chinensis). Gene. 2009;436(1-2):37-44

23. Ramak P, Kazempour Osaloo S, Ebrahimzadeh H, Sharifi M, Behmanesh M. Inhibition of the mevalonate pathway enhances carvacrol biosynthesis and DXR gene expression in shoot cultures of Satureja khuzistanica Jamzad. J Plant Physiol. 2013;170(13):1187-93.

24. Verma V, Ravindran P, Kumar PP. Plant hormone-mediated regulation of stress responses. BMC Plant Biol. 2016;16:86.

25. Leborgne-Castel N, Adam T, Bouhidel K. Endocytosis in plant-microbe interactions. Protoplasma. 2010;247(3-4):177-93.

26. Dodds PN, Rathjen JP. Plant immunity: towards an integrated view of plantpathogen interactions. Nat Rev Genet. 2010;11(8):539-48.

27. Davies PJ. The plant hormones: their nature. Occurrence, and Functions. In: Davies P.J. (eds) Plant Hormones. Dordrecht: Springer; 2010. p. 1-15. https:// doi.org/10.1007/978-1-4020-2686-7_1.

28. Kinchen JM, Ravichandran KS. Phagosome maturation: going through the acid test. Nat Rev Mol Cell Bio. 2008;9(10):781-95.

29. D'Haeze W, Holsters M. Surface polysaccharides enable bacteria to evade plant immunity. Trends Microbiol. 2004;12(12):555-61.

30. Underhill DM, Ozinsky A. Phagocytosis of microbes: complexity in action. Annu Rev Immunol. 2002;20:825-52.

31. Khraiwesh B, Zhu JK, Zhu J. Role of miRNAs and siRNAs in biotic and abiotic stress responses of plants. Biochim Biophys Acta. 2012;1819(2):137-48.

32. Flynt AS, Lai EC. Biological principles of microRNA-mediated regulation: shared themes amid diversity. Nat Rev Genet. 2008;9(11):831-42.

33. Lenka SK, Nims NE, Vongpaseuth K, Boshar RA, Roberts SC, Walker EL. Jasmonate-responsive expression of paclitaxel biosynthesis genes in Taxus cuspidata cultured cells is negatively regulated by the bHLH transcription factors TCJAMYC1, TCJAMYC2, and TCJAMYC4. Front Plant Sci. 2015;6:115. https://doi.org/10.3389/fpls.2015.00115.

34. Zhang M, Li ST, Nie L, Chen QP, Xu XP, Yu LJ, Fu CH. Two jasmonateresponsive factors, TcERF12 and TCERF15, respectively act as repressor and activator of tasy gene of taxol biosynthesis in Taxus chinensis. Plant Mol Biol. 2015;89(4-5):463-73

35. Zhang M, Chen Y, Nie L, Jin X, Liao W, Zhao S, Fu C, Yu L. Transcriptomewide identification and screening of WRKY factors involved in the regulation of taxol biosynthesis in Taxus chinensis. Sci Rep. 2018;8(1):5197.

36. Zhang $M$, Jin X, Chen Y, Wei M, Liao W, Zhao S, Fu C, Yu L. Tcmyc2a, a basic helix-loop-helix transcription factor, transduces ja-signals and regulates taxol biosynthesis in Taxus chinensis. Front Plant Sci. 2018;9:863. 
37. Rojas CM, Senthil-Kumar M, Tzin V, Mysore KS. Regulation of primary plant metabolism during plant-pathogen interactions and its contribution to plant defense. Front Plant Sci. 2014;5:17.

38. Pusztahelyi T, Holb IJ, Pocsi I. Secondary metabolites in fungus-plant interactions. Front Plant Sci. 2015;6:573.

39. Speed MP, Fenton A, Jones MG, Ruxton GD, Brockhurst MA. Coevolution can explain defensive secondary metabolite diversity in plants. New Phytol. 2015;208(4):1251-63.

40. Birkenbihl RP, Liu S, Somssich IE. Transcriptional events defining plant immune responses. Curr Opin Plant Biol. 2017;38:1-9.

41. Guo HS, Xie Q, Fei JF, Chua NH. MicroRNA directs mRNA cleavage of the transcription factor NAC1 to downregulate auxin signals for arabidopsis lateral root development. Plant Cell. 2005;17(5):1376-86.

42. Dai Z, Tan J, Zhou C, Yang X, Yang F, Zhang S, Sun S, Miao X, Shi Z. The OsmiR396-OsGRF8-OsF3H-flavonoid pathway mediates resistance to the brown planthopper in rice (Oryza sativa). Plant Biotechnol J. 2019;17(8): 1657-69.

43. Zhang $C H$, Mei XG, Liu L, Yu LJ. Enhanced paclitaxel production induced by the combination of elicitors in cell suspension cultures of Taxus chinensis. Biotechnol Lett. 2000;22(19):1561-4.

44. Martin M. Cutadapt removes adapter sequences from high-throughput sequencing reads. EMBnetjournal. 2011;17(1):10.

45. Buchfink B, Xie C, Huson DH. Fast and sensitive protein alignment using DIAMOND. Nat Methods. 2015;12(1):59-60.

46. Mortazavi A, Williams BA, McCue K, Schaeffer L, Wold B. Mapping and quantifying mammalian transcriptomes by RNA-Seq. Nat Methods. 2008:5(7): 621-8.

47. Robinson MD, McCarthy DJ, Smyth GK. edgeR: a bioconductor package for differential expression analysis of digital gene expression data. Bioinformatics. 2010;26(1):139-40.

48. Hu ZR, Liu A, Gitau MM, Huang XB, Chen L, Fu JM. Insights into the MicroRNA-regulated response of bermudagrass to cold and salt stress Environ Exp Bot. 2018:145:64-74.

49. Nystedt B, Street NR, Wetterbom A, Zuccolo A, Lin YC, Scofield DG, Vezzi F Delhomme N, Giacomello S, Alexeyenko A, et al. The Norway spruce genome sequence and conifer genome evolution. Nature. 2013;497(7451): 579-84.

50. Zhao C, Zhang G, Yin S, Li Z, Wang Q, Chen S, Zhou G. Integrated analysis of mRNA-seq and miRNA-seq reveals the potential roles of sex-biased miRNA-mRNA pairs in gonad tissue of dark sleeper (Odontobutis potamophila). BMC Genomics. 2017;18(1):613.

51. Kielbasa SM, Bluthgen N, Fahling M, Mrowka R. Targetfinder.org: a resource for systematic discovery of transcription factor target genes. Nucleic Acids Res. 2010;38(Web Server issue):W233-8.

52. Ma Z, Coruh C, Axtell MJ. Arabidopsis lyrata small RNAs: transient MIRNA and small interfering RNA loci within the Arabidopsis genus. Plant Cell. 2010;22(4):1090-103.

53. Addo-Quaye C, Miller W, Axtell MJ. CleaveLand: a pipeline for using degradome data to find cleaved small RNA targets. Bioinformatics. 2009; 25(1):130-1.

54. Dong YS, Duan WL, He HX, Su P, Zhang M, Song GH, Fu CH, Yu LJ. Enhancing taxane biosynthesis in cell suspension culture of Taxus chinensis by overexpressing the neutral/alkaline invertase gene. Process Biochem. 2015;50(4):651-60.

55. Fang ZZ, Zhou DR, Ye XF, Jiang CC, Pan SL. Identification of Candidate Anthocyanin-Related Genes by Transcriptomic Analysis of 'Furongli' Plum (Prunus salicina Lindl.) during Fruit Ripening Using RNA-Seq. Front Plant Sci. 2016;7:1338. https://doi.org/10.3389/fpls.2016.01338.

56. Dadras SS, Lin RJ, Razavi G, Kawakami A, Du J, Feige E, Milner DA, Loda MF, Granter SR, Detmar M, et al. A novel role for microphthalmia-associated transcription factor-regulated pigment epithelium-derived factor during melanoma progression. Am J Pathol. 2015;185(1):252-65.

\section{Publisher's Note}

Springer Nature remains neutral with regard to jurisdictional claims in published maps and institutional affiliations.

\section{Ready to submit your research? Choose BMC and benefit from:}

- fast, convenient online submission

- thorough peer review by experienced researchers in your field

- rapid publication on acceptance

- support for research data, including large and complex data types

- gold Open Access which fosters wider collaboration and increased citations

- maximum visibility for your research: over $100 \mathrm{M}$ website views per year

At BMC, research is always in progress.

Learn more biomedcentral.com/submissions 